\title{
The SKA contribution to GRB cosmology
}

\author{
Lorenzo Amati ${ }^{1,2}$, Alan Cosimo Ruggeri ${ }^{3,4}$, Giulia Stratta ${ }^{5}$, Salvatore Capozziello ${ }^{3,4}$, \\ Mariafelicia De Laurentis ${ }^{3,4}$, Massimo Della Valle ${ }^{2,6}$, Orlando Luongo ${ }^{3,4}$ \\ ${ }^{1}$ INAF - IASF Bologna, via P. Gobetti 101, Bologna (Italy) ${ }^{2}$ ICRANet, Piazzale della Repubblica \\ 10, Pescara (Italy) ${ }^{3}$ Università di Napoli Federico II, Via Cinthia, Napoli (Italy) ${ }^{4}$ INFN Sez. di \\ Napoli, Monte S. Angelo, Via Cinthia, Napoli (Italy) ${ }^{5}$ Universitá degli Studi di Urbino "Carlo \\ Bo", Urbino (Italy) ${ }^{6}$ INAF - Osservatorio di Capodimonte, Salita Moiariello 16, Napoli (Italy) \\ E-mail: amati@iasfbo.inaf.it
}

\begin{abstract}
Although they are not standard candles, the use of Gamma-Ray Bursts (GRBs) as a tool for measuring the geometry and expansion rate of the Universe is strongly motivated by their unique combination of huge luminosities, up to more than $10^{53} \mathrm{erg} / \mathrm{s}$, with a redshift distribution extending up to more than $z=9$. Several attempts to exploit the correlation between the photon energy at which the spectral energy distribution peaks (peak energy) and the radiated energy (or luminosity) for standardizing GRBs and to use them to estimate cosmological parameters have been made. These studies show that with the present data-set, GRBs can provide a significant and independent confirmation of $\Omega_{\mathrm{M}} \sim 0.3$ for a flat $\Lambda$ CDM. The SKA, through its unprecedented capabilities of characterizing the radio afterglow light-curve from the peak emission up to the transition to non-relativistic regime for a significant fraction of GRBs, and in combination with measurements from next generation GRB experimens, will provide unique clues on the collimation angles and energy budget of these events, thus reducing significantly the dispersion of the peak energy - radiated energy correlation and improving its reliability and accuracy in investigating dark energy properties and evolution.
\end{abstract}

Advancing Astrophysics with the Square Kilometre Array,

June 8-13, 2014

Giardini Naxos, Sicily, Italy 


\section{Introduction}

The evidence that we are living in a flat Universe undergoing accelerated expansion and in which the baryonic matter contributes only a small fraction $(\sim 4-5 \%)$ of the total matter-energy density is mostly based on combined observations of high redshift type Ia supernovae ( $\mathrm{SNe}$ Ia) and of the Cosmic Microwave Background (CMB). Other complementary probes like Galaxy Clusters and Baryonic Acoustic Oscillations (BAO) further support this scenario.

Each of these probes is affected by specific systematics, which may significantly bias the estimate of cosmological parameters, like the matter density $\Omega_{\mathrm{M}}$, the dark energy density $\Omega_{\Lambda}$ and the equation of state $(\mathrm{EoS})$ of the dark energy. More probes are now available and more crosschecks among the results obtained with each of them can be done, thus minimizing the effects of the systematics of single probes. In addition, each different class of probe has a different "sensitivity" to cosmological parameters depending on its redshift distribution. Thus, the search for more classes of sources that can be used as standard candles to study the geometry and the evolution of the expansion rate of the Universe is highly motivated.

With an observed redshift distribution extending from 0.0085 (GRB 980425) up to 8.26 (GRB 090423) or $\sim 9.2$ if we include photometric redshift (GRB 090429B) and isotropic-equivalent radiated energies up to more than $\sim 10^{54} \mathrm{erg}$, released typically in a few tens or hundreds of seconds, Gamma-Ray Bursts (GRBs) are the brightest cosmological sources in the Universe (Fig. 1). In addition, they emit most of their radiation in the hard X-rays, thus, contrary to SNe Ia, they are not affected by dust absorption. Unfortunately, they are not standard candles, given that their radiated energies, or luminosities, span several orders of magnitude. However, as we discuss in the next Sections, the evidence for a strong correlation between the photon energy at which the spectral energy distribution (SED) peaks and the GRB intensity has opened the possibility of using them as cosmological probes.

\section{The correlation between GRB spectral peak energy and intensity.}

GRBs show non thermal spectra which can be modeled with broken power laws with 3 pa-
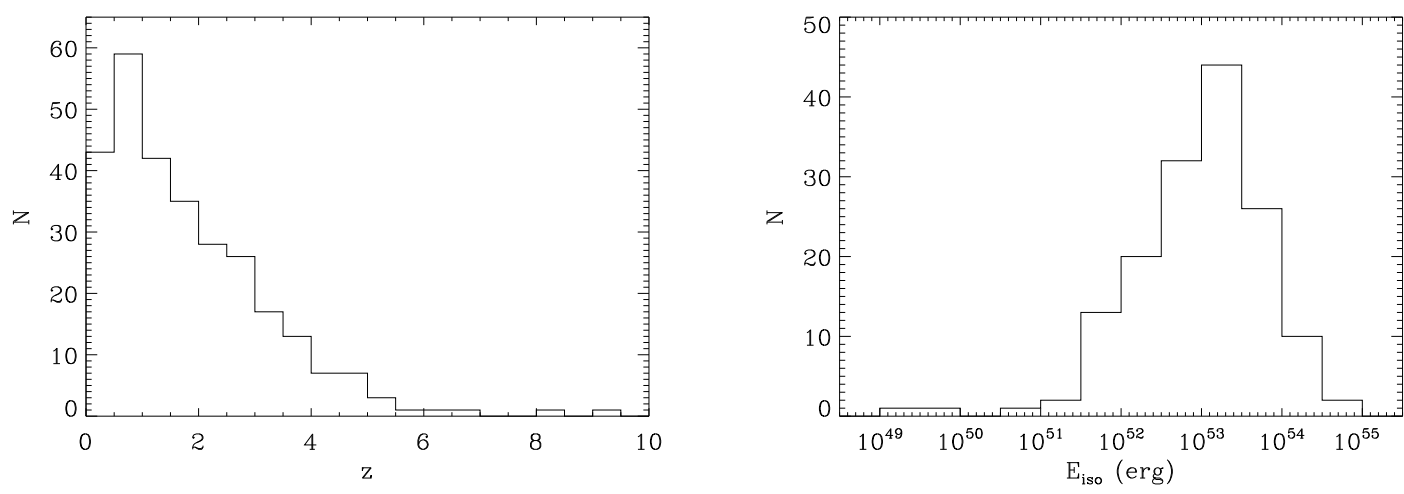

Figure 1: Distributions of redshift and isotropic-equivalent radiated energy, $E_{\text {iso }}$, of long GRBs (as of end of 2013). 
rameters: the low-energy spectral index $\alpha$, the high energy spectral index $\beta$ and the "roll-over" energy $E_{0}$. The peak of the spectral energy distribution (Fig. 2) is a characteristic quantity in GRB emission models and is called peak energy, $E_{\mathrm{p}}$. Converting the observed spectrum into a rest-frame spectrum via optical redshift measurements for a small fraction of GRBs has enabled the estimate of two key intrinsic quantities: the total radiated energy computed by integrating the spectrum in a standard (1-10000 keV) energy band and assuming isotropic emission, $E_{\text {iso }}$, and the peak energy of the cosmological rest-frame $v F_{v}$ spectra of GRBs, $E_{\mathrm{p}, \mathrm{i}}=E_{\mathrm{p}} \times(1+z)$. As of the end of 2013, the number of GRBs with measurements of the redshift and of the spectral parameters accurate enough to allow a reliable computation of $E_{\mathrm{p}, \mathrm{i}}$ and $E_{\mathrm{iso}}$, is $\sim 160$. The distribution of $E_{\mathrm{p}, \mathrm{i}}$ for these events is approximately a Gaussian centered at a few hundreds of $\mathrm{keV}$ and with a low energy extension down to a few keV, corresponding to the so-called X-Ray Flashes (XRFs) or X-Ray Rich (XRR) events. Whether this distribution is significantly biased by instrumental effects or is an intrinsic properties of GRBs is still unclear, in particular concerning the real fraction of XRFs and XRRs with respect to "normal GRBs". The distribution of $E_{\text {iso }}$ is somewhat similar, extending from $\sim 10^{51}$ to more than $\sim 10^{54} \mathrm{erg}$, peaking at $\sim 10^{53} \mathrm{erg}$ and with a few peculiar weak events $\left(\sim 10^{48}-\sim 10^{50} \mathrm{erg}\right)$.

In 2002, based on a small sample of BeppoSAX GRBs with known redshift and spectral parameters, it was discovered (Amati et al., 2002) that $E_{\mathrm{p}, \mathrm{i}}$ is significantly correlated with $E_{\mathrm{iso}}$. This correlation has the form

$$
\log E_{\mathrm{p}, \mathrm{i}}(\mathrm{keV})=m \log E_{\mathrm{iso}}\left(10^{52} \mathrm{erg}\right)+q,
$$

with $m \sim 0.5$ and $q \sim 2$, and is characterized by an extra-Poissonian scatter normally distributed with a $\sigma_{\text {ext }}$ of $\sim 0.2$ dex around the best-fit law. Subsequent observations with various detectors and spectrometers confirmed and extended the $E_{\mathrm{p}, \mathrm{i}}-E_{\text {iso }}$ correlation, showing that it holds for all long GRBs and XRFs with well measured redshift and spectral parameters (Amati, 2006; Amati et al., 2008). The present distribution of long GRBs and XRFs in the $E_{\mathrm{p}, \mathrm{i}}-E_{\text {iso }}$ plane is shown in Fig. 2 (see caption for details). The existence, properties (slope and dispersion) and extension over several orders of magnitude in both $E_{\mathrm{p}, \mathrm{i}}$ and $E_{\mathrm{iso}}$ of the correlation provide observational challenges for the models of the physics and geometry of the prompt emission of GRBs (e.g., Zhang \& Mészáros, 2002; Lamb et al., 2004; Amati, 2006).

\section{GRB cosmology through the $E_{\mathrm{p}, \mathrm{i}}$-intensity correlation}

Since the $E_{\mathrm{p}, \mathrm{i}}-E_{\text {iso }}$ correlation correlates the quantity $E_{\mathrm{p}, \mathrm{i}}$ (which is independent of cosmological model), with the quantity $E_{\text {iso }}$ (whose computation requires the luminosity distance $D_{L}$ ), then it could theoretically be used to "standardize GRBs", in a way similar to the use of the Phillips relation (Phillips, 1993) to standardize SNe Ia. However, the scatter of the correlation (Amati et al., 2008) and the lack of a sufficient number of very low redshift GRBs allowing a cosmologicalindependent calibration has been a challenge to its use for this purpose.

The investigations of GRBs as cosmological probes started in 2004, when it was found that the $E_{\mathrm{p}, \mathrm{i}}-E_{\mathrm{iso}}$ correlation tightens by substituting $E_{\text {iso }}$ with the collimation-corrected radiated energy $E_{\gamma}=\left(1-\cos \theta_{j e t}\right) \times E_{\text {iso }}$ (Ghirlanda et al., 2004; Dai et al., 2004). This result was based on a small sub-sample of GRBs with known $E_{\mathrm{p}, \mathrm{i}}$ and $E_{\text {iso }}$ for which it was possible to infer the jet opening angle $\theta_{\text {jet }}$ from the break time, $\mathrm{t}_{\mathrm{b}}$, at which the decay index of the light curve of the optical 

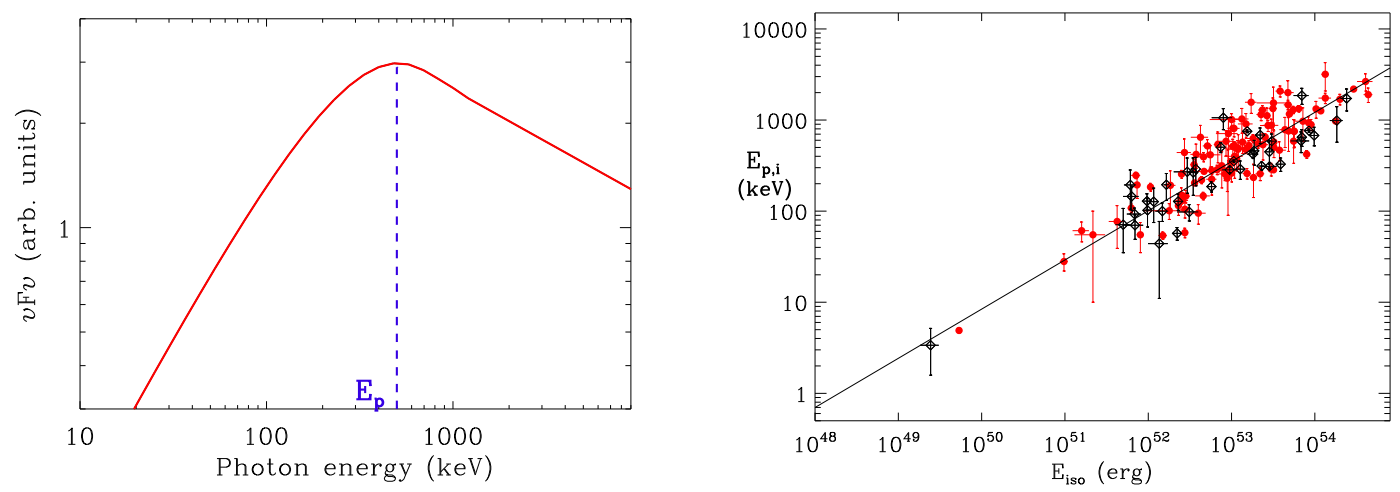

Figure 2: Left: typical $v F_{v}$ (energy) spectrum of a GRB in its cosmological rest-frame. Right: the $E_{\mathrm{p}, \mathrm{i}}-$ $E_{\text {iso }}$ correlation in long GRBs (as of the end of 2013). The black line shows the best-fit power law. Data from Amati et al. (2008), Amati et al. (2009) and citetAmdv13. These values are based on spectral data and fluences reported in official catalogs and in the GCN Circulars (gcn.gsfc.nasa.gov/gcn3_archive.html) by following the method outlined, e.g., in Amati et al. (2002) and Amati (2006).

afterglow sharply changes to a steeper one, under the assumption of a simple jet and afterglow model (Sari et al., 1999). With this improvement it was possible to derive, within the standard $\Lambda \mathrm{CDM}$ cosmological model, estimates of $\Omega_{\mathrm{M}}$ and $\Omega_{\Lambda}$ consistent with the "concordance" values mostly coming from the analysis of SNe Ia and the CMB (see review by Ghirlanda et al. (2006)). These results prompted other studies aimed at deriving a GRB Hubble diagram, based, e.g., on the joint use of the $E_{\mathrm{p}, \mathrm{i}}-E_{\gamma}$ correlation together with other weaker correlations between luminosity and observed properties (Schaefer, 2007), or the calibration of the $E_{\mathrm{p}, \mathrm{i}}-E_{\gamma}$ correlation with $\mathrm{SNe}$ Ia (e.g., Kodama et al., 2008; Demianski et al., 2008). In recent years, the simple jet model assumed to compute $\theta_{j e t}$ from the break time of the optical afterglow light curve has been seriously challenged by the observation that in several cases the the X-ray afterglow light curves show no jet-break or show a break not simultaneous to the optical one. This makes the determination of $E_{\gamma}$, and thus the characterization and use of the $E_{\mathrm{p}, \mathrm{i}}-E_{\gamma}$ correlation, less firm.

This uncertainty has led to a re-evaluation of the simple $E_{\mathrm{p}, \mathrm{i}}-E_{\mathrm{iso}}$ correlation as a cosmological tool, as it has many advantages, e.g., a sample size four times larger, is based on only two variables, and avoids model-dependent assumptions (Amati et al., 2008). By assuming a flat $\Lambda$ CDM Universe, the $\chi^{2}$ obtained by fitting this correlation with a simple power law is a function of the value of $\Omega_{M}$ assumed in the computation of $E_{\text {iso }}{ }^{1}$ In particular, as can be seen in Fig. 3, the function $\chi^{2}\left(\Omega_{\mathrm{M}}\right)$ shows a parabolic shape with a minimum at $\Omega_{\mathrm{M}} \sim 0.25-0.30$. This is a simple but relevant result: a) it shows that the $E_{\mathrm{p}, \mathrm{i}}-E_{\text {iso }}$ correlation can indeed be used to extract information on cosmological parameters; b) it provides evidence, independently from SNe Ia or other probes, that, if we live in a flat Universe as predicted by many inflationary models and supported by CMB measurements, the density of the Universe is dominated by an unknown component (e.g., dark energy).

The simple $\chi^{2}$ method cannot be used to obtain reliable confidence levels on both the parameters of the correlation (normalization and slope) and the cosmological parameters. Instead a

\footnotetext{
${ }^{1}$ This result was originally based on the sample of 70 long GRBs with known $E_{\mathrm{p}, \mathrm{i}}$ and $E_{\mathrm{iso}}$ published by Amati et al. (2008).
} 

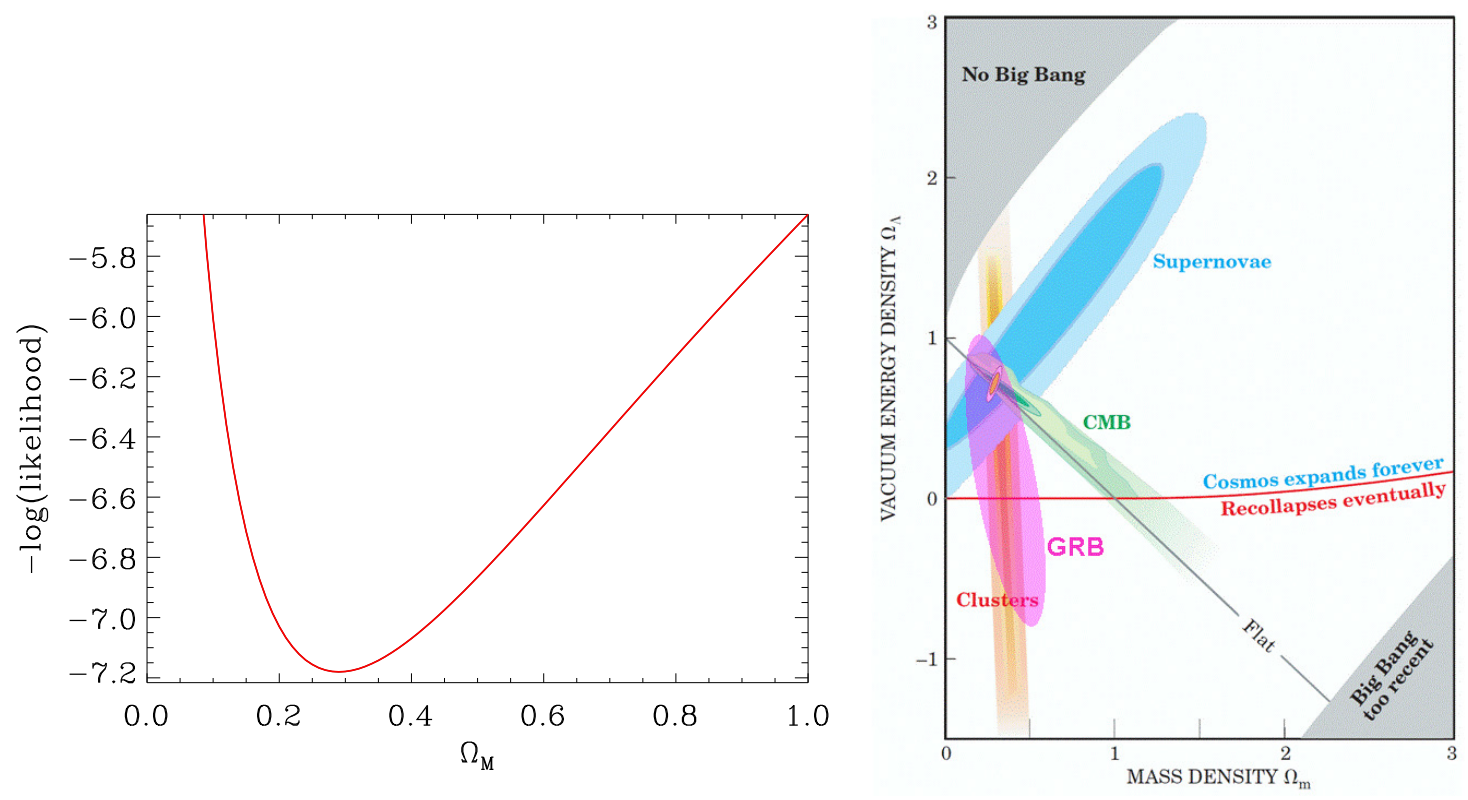

Figure 3: Left: goodness of fit of the $E_{\mathrm{p}, \mathrm{i}}-E_{\mathrm{iso}}$ correlation of long GRBs, expressed in terms of $-\log$ (likelihood), as a function of the value of $\Omega_{\mathrm{M}}$ used to compute the $E_{\text {iso }}$ values in a standard $\Lambda$ CDM flat universe (for a sample of 156 GRBs available as of the end of 2012). Right: $68 \%$ confidence level contour in the $\Omega_{\mathrm{M}}-\Omega_{\Lambda}$ plane obtained by releasing the flat Universe assumption and assuming a sample of sample of 250 GRBs expected in the near future (see Table 1 and text) compared to those from other cosmological probes (adapted from a figure by the Supernova Cosmology Project; Suzuki et al. 2012). From Amati \& Della Valle (2013).

maximum likelihood method accounting for the uncertainties on both the $X$ and $Y$ quantities and the extra variance $\sigma$ ext is adopted (Amati et al., 2008). In Table 1, we show the $68 \%$ and $90 \%$ confidence level intervals for $\Omega_{\mathrm{M}}$, in the assumption of a flat Universe, obtained in this way with samples of 70 GRBs (Amati et al., 2008) and 156 GRBs (Amati \& della Valle, 2013). As can be seen, by increasing the number of GRBs in the sample the accuracy of the estimate of $\Omega_{\mathrm{M}}$ improves. This is confirmed also by the investigation of the confidence contours in the $\Omega_{M}-\Omega_{\Lambda}$ plane computed by following the same method as above but releasing the flat Universe assumption (Fig. 3.).

Broad energy band spectroscopy of GRBs (from $\sim 10-15 \mathrm{keV}$ up to several MeVs) is required to obtain reliable estimates of $E_{\mathrm{p}, \mathrm{i}}$ and $E_{\mathrm{iso}}$. The main contribution to the number of GRBs that populate the $E_{\mathrm{p}, \mathrm{i}}-E_{\text {iso }}$ plane comes from joint detections by Swift the Fermi/GBM and/or KonusWIND. Hopefully, these missions will continue to operate for at least a few years, providing $\sim 15-$ $20 \mathrm{GRBs} / \mathrm{year}$, to increase the $E_{\mathrm{p}, \mathrm{i}}-E_{\mathrm{iso}}$ sample and refine the present estimates of cosmological parameters (Fig. 3 and Table 1). A further improvement should come from next generation (after 2018) missions capable of performing both the task of prompt accurate localization and broadband spectroscopy of GRBs, like the French-Chinese Space Variable Objects Monitor (SVOM) or the proposed Ultra-Fast Flash Observatory (UFFO) mission, an international project led by Korea and Taiwan. 
Table 1: Comparison of the $68 \%$ confidence intervals on $\Omega_{\mathrm{M}}$ and $w_{0}\left(\Omega_{\mathrm{M}}=0.3, w_{\mathrm{a}}=0.5\right.$; a standard parametrization of the dark energy equation of state: $w=w_{0}+w_{\mathrm{a}} z /(1+z)$ was assumed) for a flat FriedmannLemaître-Robertson-Walker (FLRW) universe obtained with the sample of 70 GRBs by Amati et al. (2008), the sample of 156 GRBs available as of the end of 2012 (Amati \& della Valle, 2013) and simulated samples of 250 and 500 GRBs (see text). In the last three lines we also show the results obtained for the same samples by assuming that the dispersion of the correlation between $E_{\mathrm{p}, \mathrm{i}}$ and radiated energy is reduced by a factor of 2 thanks to the measurements by the SKA allowing inferences on the kinetic energy and collimation angle of the emitting plasma.

\begin{tabular}{lcc}
\hline GRB \# & $\begin{array}{c}\Omega_{\mathrm{M}} \\
\text { (flat) }\end{array}$ & $\begin{array}{c}w_{0} \\
\left.\text { (flat, } \Omega_{\mathrm{M}}=0.3, w_{\mathrm{a}}=0.5\right)\end{array}$ \\
\hline 70 (real) GRBs (Amati et al., 2008) & $0.27_{-0.18}^{+0.38}$ & $<-0.3(90 \%)$ \\
156 (real) GRBs (Amati \& della Valle, 2013) & $0.29_{-0.15}^{+0.28}$ & $-0.9_{-1.5}^{+0.4}$ \\
250 (156 real + 94 simulated) GRBs & $0.29_{-0.12}^{+0.16}$ & $-0.9_{-1.1}^{+0.3}$ \\
500 (156 real + 344 simulated) GRBs & $0.29_{-0.09}^{+0.10}$ & $-0.9_{-0.8}^{+0.2}$ \\
\hline 156 (real) GRBs, SKA & $0.30_{-0.06}^{+0.06}$ & $-1.1_{-0.30}^{+0.25}$ \\
250 (156 real + 94 simulated) GRBs, SKA & $0.30_{-0.05}^{+0.04}$ & $-1.1_{-0.20}^{+0.20}$ \\
500 (156 real + 344 simulated) GRBs, SKA & $0.30_{-0.03}^{+0.03}$ & $-1.1_{-0.15}^{+0.12}$ \\
\hline
\end{tabular}

\section{Improving GRB cosmology with the SKA}

The improvement in the accuracy of the estimate of cosmological parameters with the $E_{\mathrm{p}, \mathrm{i}}-$ $E_{\text {iso }}$ correlation obtained by enlarging the sample on which the analysis is performed (Tab. 1), confirms that the method is reliable and promising. However, a real breakthrough in these field would be obtained if the dispersion of the correlation between $E_{\mathrm{p}, \mathrm{i}}$ and radiated energy or luminosity could be reduced by at least a factor of 2 (e.g., Ghirlanda et al. 2004). In particular, the accuracy in the estimate of cosmological parameters and dark energy properties with GRBs is presently limited by the large uncertainties affecting our knowledge of the truly radiated energy, which forces us to use the isotropic-equivalent quantity $E_{\text {iso }}$. This conservative assumption, while accounting for our ignorance on the true energy budget, is expected to contribute at least $50 \%$ of the correlation dispersion. In this section we show that, in this respect, the radio measurements that will be provided initially by the SKA1-MID and then by the full SKA2 will be fundamental. Indeed, as detailed below, the measurements of the radio afterglow emission that the SKA will perform for most of the GRBs detected and localized at high energies will allow to infer both the jet opening angle and the fireball kinetic energy. As we are going to discuss, these quantities enable to obtain more accurate estimates of the truly radiated energy with respect to $E_{\text {iso }}$, thus substantially reducing the dispersion of the radiated energy $-E_{\mathrm{p}, \mathrm{i}}$ correlation and improving its reliability and power for cosmology.

\subsection{Radio emission from GRBs: perspectives with the SKA}

Accretion onto a black hole formed during a GRB event produces two jets along which matter is ejected at relativistic velocities (fireball). Part of the fireball kinetic energy is converted into electromagnetic (synchrotron) radiation producing the afterglow emission while the ejecta gradually slows down to a non-relativistic regime (e.g. Panaitescu, 1999). The photon energy corresponding 


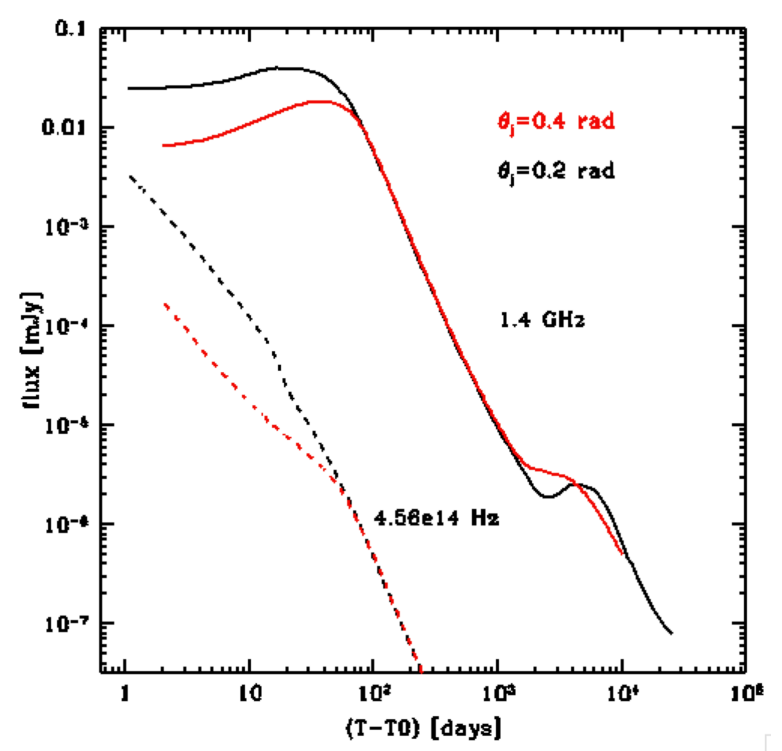

Figure 4: Simulated afterglow light curves in radio (solid line) and optical (dashed line) bands from Van Eerten et al. (2010), obtained assuming two possible jet opening angle $\left(\theta_{j e t}=0.2\right.$ radian plotted in red, and $\theta_{j e t}=0.4$ radian plotted in black). The light curves clearly show different behaviour up to about tens of days after the trigger in both energy bands depending on the jet opening angle. The simulation assumes a GRB at a luminosity distance of $10^{28} \mathrm{~cm}$, with energy $10^{50} \mathrm{erg}$, and a surrounding interstellar matter density of $10^{-3} \mathrm{~cm}^{-3}$.

to peak of the spectral energy distribution evolves with time towards longer wavelengths and at days up to months from the burst onset (depending on the frequency), it is expected to appear at radio frequencies.

In the Swift era, radio afterglow emission is being detected only for about $30 \%$ of accurately ( $<$ a few arcmin) localized GRBs, compared with $\sim 93 \%$ in X-rays and $\sim 75 \%$ in optical/NIR. Most measurements have been made with the Very Large Array (VLA) and Expanded Very Large Array (EVLA), although other telescopes have contributed (Westerbork Synthesis Radio Telescope (WSRT), the Australia Telescope Compact Array (ATCA), the Giant Metrewave Radio Telescope (GMRT), a few by the Very Long Baseline Array (VLBA)). The canonical long-duration GRB radio light curve at $8.5 \mathrm{GHz}$ peaks at three to six days in the source rest frame, with a median peak luminosity of $10^{31} \mathrm{erg} \mathrm{s}^{-1} \mathrm{~Hz}^{-1}$. The typical mean fluxes at $8.5 \mathrm{GHz}$ in 5-10 days from the GRB range from $\sim 100$ to $\sim 900 \mu \mathrm{Jy}$. Peak fluxes may occasionally reach $10 \mathrm{mJy}$. See, e.g., Chandra \& Frail (2012) and Granot \& Van del Horst (2014) for a review.

As discussed, e.g., by Chandra \& Frail (2012), the small range of peak flux densities between the radio detection and non-detections suggests that the detection fraction of radio afterglows is largely determinated by the instrumental sensitivities. The SKA, already in its Phase 1, will shed light on this fundamental issue, given that the current upper limits set by VLA and EVLA observations are of the order of a few tens of $\mu \mathrm{Jy}(8.5 \mathrm{GHz})$ and the foreseen SKA1-MID continuum sensitivity is of the order of $0.72 \mu \mathrm{Jy}_{-}-\mathrm{hr}^{-1 / 2}$ (Dewdney et al., 2013).

GRB population synthesis codes show that all GRBs with gamma-ray peak flux above the 
Swift/BAT flux threshold $\left(0.4 \mathrm{ph} \mathrm{cm}^{-2} \mathrm{~s}^{-1}\right)$ have a peak flux in radio above $1 \mu \mathrm{Jy}$, thus already detectable by the SKA Phase 1. The major difference between present/forthcoming SKA precursors and the final SKA can be appreciated considering the entire population of GRBs, thus including those GRBs with gamma-ray flux well below the present detector sensitivity. For example, it is foreseen that the SKA will be able to detect at radio frequencies all the bursts, with a detection rate of $480 \mathrm{GRBs} \mathrm{yr}^{-1} \mathrm{sr}^{-1}$ (Ghirlanda et al., 2013).

The combination of high sensitivity, high angular resolution, short reaction time and broad band of the SKA (initially with the SKA1 and then with the full SKA) will allow to achieve important and partly unprecedented goals for GRB science. These include: measurements of GRB source size and expansion velocities through ISM scintillation; accurate localization of GRBs in host galaxies; measurement of host galaxy radio emission; early radio afterglow: physics (reverse shock, transition from optically thick to optically thin synchrotron emission, etc.), estimate of kinetic energy and jet opening angle from the SED and light curve fitting. In the next sections we show how the two latters measurements are fundamental for the role of GRBs in cosmology.

\subsection{Impact on measuring cosmological parameters with GRBs}

Concerning GRB cosmology, the high sensitivity of the SKA will allow accurate radio calorimetry for a large number of GRBs. In the following sections we show how a significant improvement in using GRBs to measure cosmological parameters would come from a more reliable way to estimate the jet opening angle and, thus, the true radiated energy and/or the kinetic energy of the fireball in non-relativistic regime. Only observations of a large number of radio afterglows offer this opportunity.

\subsubsection{SKA Phase 1: measuring the jet collimation angle}

In the simplest modelling of the afterglow light curve, after reaching its maximum intensity, the radiative flux decays following a power law function with decay index $\sim 1$. "Achromatic" steepening (i.e. a steepening not dependent from the photon energy) to a decay index $\sim 2.2$ is expected when the relativistic collimation angle $1 / \Gamma(t)$ within which the expanding fireball radiates, becomes greater than jet collimation angle $\theta_{j}$ because of the fireball deceleration when interacting with the ISM (Sari et al., 1998; Sari \& Piran, 1999). The achromatic break epoch (jet break) observed in the light curve is a function of the jet aperture angle, thus measuring this time it is possible to inferr $\theta_{j}$. However, as mentioned already in Section 3, in the last years this simple jet model has been seriously challenged by the observed different behavior of the X-ray afterglow light curves, which, in several cases, show no jet break or show a break not simultaneous to the optical one. This makes the determination of $\theta_{j}$ through observations of jet breaks uncertain in several cases. In addition, for jet opening angles larger than few degrees, achromatic jet breaks are expected days or weeks after the trigger when the optical and X-ray afterglow are typically well below the instrumental detection thresholds.

A more promising method to estimate $\theta_{j}$ may reside in the comparison of the observed multiwavelength light curves with synthetic light curves obtained from relativistic hydrodynamical simulations (e.g. Van Eerten et al. 2010). The simulated light curves represent a more realistic description of the observed afterglow phenomenology. In Figure 4 is shown an example of synthetic light curve of the optical and radio afterglows, obtained from relativistic hydrodinamic simulations 

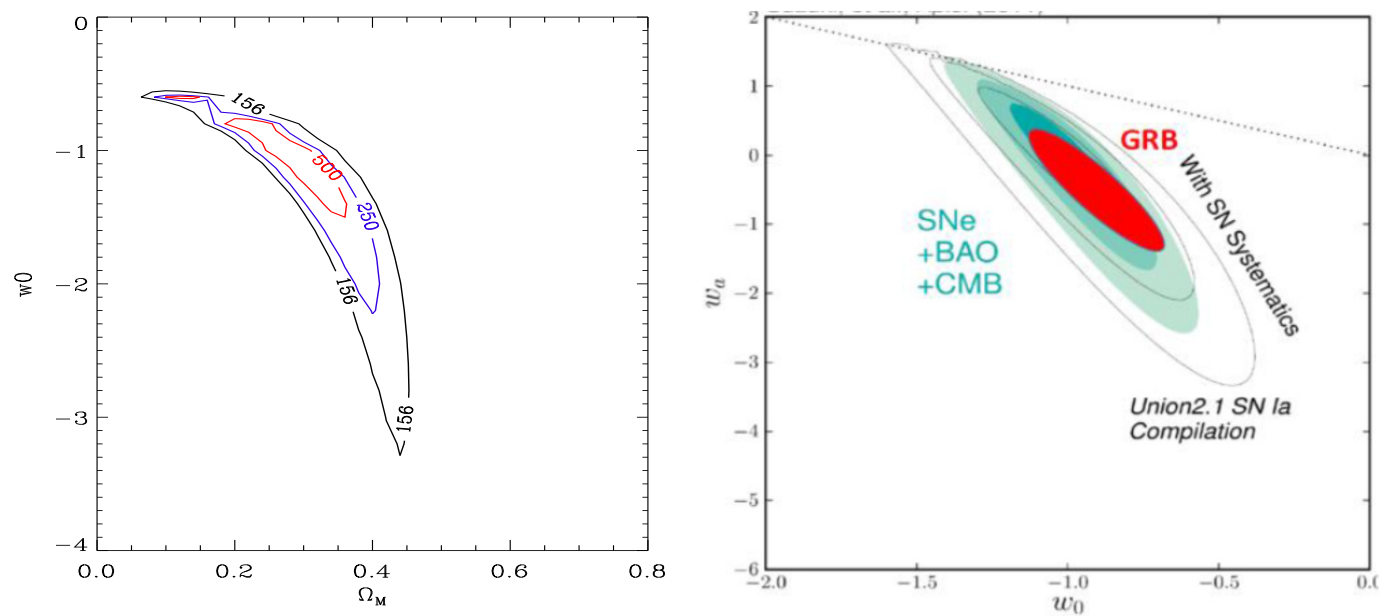

Figure 5: $68 \%$ confidence level contours in the $\Omega_{\mathrm{M}}-w_{0}$ and $w_{0}-w_{\mathrm{a}}$ planes (by assuming a standard parametrization of the dark energy equation of state: $\left.w=w_{0}+w_{\mathrm{a}} z /(1+z)\right)$ for a flat FLRW universe derived from the expected future samples by assuming that the scatter of the $E_{\mathrm{p}, \mathrm{i}}$ - radiated energy correlation is reduced by a factor of 2 thanks to the measurements by the SKA. In the right panel, we show only the contour obtainable with $500 \mathrm{GRBs}$, compared to those form other cosmological probes combined together (adapted from Suzuki et al. 2012).

joined with radiative transfer code (van Eerten et al. 2010) of two GRBs with identical energetics, ISM densities and distances, but with two different jet opening angles. The plot clearly show how the overall behaviour of the light curve depends on the jet opening angle and thus can constrain it by comparison with observations.

With a sensitivity of $\sim 1 \mu \mathrm{Jy}$, almost all the GRB detected today with Swift could be not only detected but monitored in time for days up to weeks already with the SKA in Phase 1 (see for example Figure 4 in Ghirlanda et al. 2013) thus complementing the multi-wavelength picture of the afterglow and, by comparison with synthetic light curves, constraining the jet opening angle and ultimately the true radiated energy budget $E=E_{i s o}\left(1-\cos \theta_{j}\right)$.

\subsubsection{SKA Phase 2: measuring the fireball kinetic energy}

Another more challenging way to estimate the radiated energy content of GRBs relies on late time monitoring. At about 100 days after the burst (but theoretical predictions are still compatible with a range that goes from 10 to 1000 days), the decelerating fireball is expected to reach a non-relativistic expansion velocity (Livio \& Waxman, 2000) and an isotropic geometry due to the increasing lateral spreading of the jet (Frail et al., 2000). Radiation at this time is expected only in the radio frequencies. Radio emission marks this transition with an expected flattening of its power law decaying light curve. The non-relativistic regime transition epoch, $t_{N R}$, depends from the fireball kinetic energy $E_{K}$ (Frail et al., 2001) that consequentely can be derived once $t_{N R}$ has been measured. Therefore, by assuming a kinetic-to-radiated energy conversion efficiency $\eta$, from the kinetic energy it is possible to estimate the true radiated energy in an independent way from the collimation angle estimate as $E=\eta E_{K}$. In those cases where $\theta_{j}$ is also known, together with $E_{K}$, a complete picture can be obtained by estimating the conversion efficiency $\eta$. 
Flux levels at $t_{N R}$ are expected to be $<10 \mu \mathrm{Jy}$. While SKA in Phase 1 is expected to measure $t_{N R}$ for about $30 \%$ of the Swift/BAT GRBs sample, this number will increase to about $70 \%$ with the SKA at the end of Phase 2 assuming a 10-fold improved sensitivity (see for example Figure 5 in Ghirlanda et al. 2013).

\subsubsection{Expected accuracy for dark energy investigation}

The strong impact of the reduction of the dispersion of the $E_{\mathrm{p}, \mathrm{i}}-E_{\mathrm{iso}}$ correlation by a factor of $\sim 2$ thanks to the estimate of the jet opening angle and, possibly, the kinetic energy of the blastwave, on the accuracy in the measurement of cosmological parameters with GRBs can be seen by comparing the first and second part of Table 1 (see also the simulations performed by Ghirlanda et al. 2004). Of particular interest is the perspective capability of GRBs of contributing to the characterization of dark energy and its evolution, one of the most relevant open issues in modern science, as illustrated in Figure 5. The start of the SKA Phase 1 early science will coincide with the launch of the Chinese-French SVOM space mission dedicated to GRBs (Godet et al., 2012). This mission, combined with the follow-up observations by the large optical and IR facilities that will be operating at that epoch, is expected to provide us with the measure of redshift and spectral peak energy (the basic information required to use a GRB for cosmology through the $E_{\mathrm{p}, \mathrm{i}}$ - $E_{\text {iso }}$ correlation) for about $50 \mathrm{GRBs} /$ year. Based on the SKA phase 1 capabilities (early science and nominal) summarized above and by taking into account that the sensitivity of SVOM will be worse with respect to that of Swift/BAT (i.e. it will detect brighter GRBs), we expect that SKA1, already in its early science phase, will be capable of detecting and characterizing the radio afterglow emission of most SVOM GRBs with measured redshift and peak energy. By combining the SKA measurements with those at other wavelengths, it will then be possible, as discussed in previous section, to get a reliable estimate of the true radiated energy of $40-50$ GRB year $^{-1}$. This means that the accuracy on the estimates of $\Omega_{\mathrm{M}}, \Omega_{\Lambda}$ and on the parameters of the equation of state of dark energy reported in Table 1 and Figure 5 for 156, 250 and 500 GRBs will be reachable in about 3,5 and 10 years of operation of SKA Phase 1. Actually, such results may be obtained even in shorter times, if we take into account that, in addition to SVOM, it is likely that other satellites with GRB detection and location capabilities will be flying in the next decade (several projects are under study and being proposed).

As can be inferred from the expected performances summarized in previous subsection, the coming of operative SKA Phase 2 at the end of next decade, combined with large GRB facilities with sensitivities significantly better than Swift and SVOM and autonomous optical / IR follow-up capabilities (e.g., as proposed to ESA, Amati et al. 2013), may provide us with accurate measurements of redshift, spectral peak energy, radio afterglow light curve and kinetic energy for hundreds of GRBs in a few years, thus pushing the investigation of dark energy with GRBs at its extreme (e.g., the study of the evolutionary properties of dark energy shown in the right panel of Fig. 5 may be obtained in 8-10 years of the SKA Phase 1 and $\sim 3$ years of the SKA Phase 2).

\section{Summary and conclusions}

Given their huge radiated energies and redshift distribution extending from $\sim 0.01$ up to 9.2, GRBs are potentially a very powerful cosmological probe, complementary to other probes (e.g., 
SNe Ia, galaxy clusters, BAO). As demonstrated by several works, the most promising tool allowing the use of GRBs for measuring cosmological parameters and, in perspective, characterizing the properties of "dark energy" is the correlation between the burst radiated energy $E_{\text {iso }}$ and the spectral peak energy $E_{\mathrm{p}, \mathrm{i}}$. The main limit of the use of the $E_{\mathrm{p}, \mathrm{i}}-E_{\mathrm{iso}}$ correlation in cosmology so far comes from its large scatter, that is in part due to our ignorance on the emission processes at play during a burst and partly to the unknown collimation factor of the ejecta (forcing us to use the isotropic emission approximation). Radio observations with the SKA Phase 1 and the full SKA (Phase 2) will provide an important step forward in the attempt of estimating the true radiated energy of GRBs and, hence, reducing significantly the dispersion of the correlation between $E_{\mathrm{p}, \mathrm{i}}$ and radiated energy (or luminosity). More specifically, with SKA Phase 1 this goal will be mostly achieved by getting reliable estimates of the jet opening angle, and therefore of the collimation corrected radiated energy, for a large sample of GRBs, through the monitoring the afterglow flux evolution with time from the early up to late epochs and by comparing it with simultaneous multiwavelength observations and synthetic light curves. With the Phase 2 of SKA there will be also the possibility of estimating, for about $50 \%$ of the GRBs detectable by space detectors, the true energy budget through the fireball kinetic energy (from which $E=\eta \times E_{K}$ where $\eta$ is assumed to be typically 0.1 ) by very late epochs radio monitoring of the fireball in its non-relativistically expanding regime. Simultaneous observations from high-energy satellites expected to be operative at the time the SKA will reach its full sensitivity, will continue to provide measures of the burst spectrum peak energy. As supported by previous studies (e.g., Ghirlanda et al., 2004) and simulations here reported, the expected reduction of the correlation scatter by using a better proxy to the true radiated energy of GRBs (Ghirlanda et al., 2004) will provide substantial improvement in the accuracy of the estimate of cosmological parameters with GRBs.

\section{References}

Amati, L., Frontera, F., Tavani, M., et al., 2002, A\&A, 390, 81

Amati, L., 2006, MNRAS, 372, 233

Amati, L., Della Valle, M., Frontera F. et al., 2007, A\&A, 463, 913

Amati, L., Guidorzi, C., Frontera F. et al., 2008, MNRAS, 391, 577

Amati, L., Frontera, F. \& Guidorzi, C., 2009, A\&A, 508, 173

Amati, L. \& Della Valle, M., 2013, IJMPD, 22, 1330028

Burlon, D., Ghirlanda, G., van der Horst, A., et al., 2015, "The SKA View of Gamma-Ray Bursts", in "Advancing Astrophysics with the Square Kilometre Array", Proceedings of Science, PoS(AASKA14)052 (arXiv:1501.04629)

Chandra, P. \& Frail, D.A., 2012, ApJ, 746, 156

Dai, Z.G., Liang, E.W. \& Xu, D., 2004, ApJ, 612, L101

Demianski, M., Piedipalumbo, E., Rubano, C, Scodellaro, P., 2008, A\&A, 481, 279

Dewdney, P., Turner, W., Millenaar, R., McCool, R., Lazio, J., Cornwell, T., 2013, "SKA1 System Baseline Design", Document number SKA-TEL-SKO-DD-001 Revision 1

Frail, D. A., Waxman, E. and Kulkarni, S.R., 2000, ApJ, 537, 191

Frail, D. A., et al., 2001, ApJ, 562, L55 
Ghirlanda, G., Ghisellini, G., Lazzati, D., 2004, ApJ, 616, 331 Gamma-Ray Burst Energies Correlate with the Peak Energy of Their $v F_{v}$ Spectrum

Ghirlanda, G., Ghisellini, G., Firmani, 2006, New J. Phys., 8, 123 candles to constrain the cosmological parameters

Ghirlanda, G., Salvaterra, R., Burlon, D. et al., 2013, MNRAS, 435, 2543

Godet, O., Paul, J., Wei ,J.Y., et al., 2012, SPIE Conf. Ser. 8443, p. 1

Granot, J. \& van der Horst, A. J., 2014, PASA, 31, 8

Kodama, Y., Yonetoku, D., Murakami, T. et al., 2008, MNRAS, 391, L1

Lamb, D. Q., Ricker, G. R., Atteia, J.-L. et al., 2004 NewAR, 48, 423

Livio, M \& Waxman, E., 2000, ApJ, 538, 187

Panaitescu, A. and Meszaros, P. 1999, ApJ, 526, 707

Phillips, M.M., 1993, ApJ, 413, L105

Sari, R., Piran, T., \& Narayan, R., 1998, ApJ, 497, L17

Sari R. \& Piran, T. 1999 ApJ, 520, L641

Sari, R., Piran, T. \& Halpern, J.P., 1999, ApJ, 519, L17

Schaefer, B.E., 2007, ApJ, 660, 16

Suzuki, N., Rubin, D., Lidman, C. et al., 2012, ApJ, 746, 85

Zhang, B. \& Mészáros, P., 2002, ApJ, 581, 1236 